\title{
Polihedral Küme-Değerli Dönüşümlerin Yardımıyla Bir Eşitsizlik Sisteminin Çözülebilirliği Üzerine
}

\author{
On the Solvability of an Inequality System via Polyhedral Convex Set-Valued Mappings
}

\author{
Özkan DEĞER ${ }^{1}$ \\ ${ }^{1}$ İstanbul Üniversitesi, Fen Fakültesi, Matematik Bölümü, Vezneciler, 34134, İstanbul, Türkiye
}

$\ddot{O} \mathbf{z}$

Küme-değerli dönüşümlerin bilimsel, teknik ve diğer akademik disiplinlerdeki çeşitli çalışmalarda ortaya çıkan problemlerin çözümünde temel bir matematiksel araç olarak kullanılması günden güne hızla artmaktadır. Örneğin doğrusal olmayan analiz, doğrusal olmayan programlama, matematiksel ekonomi ve işletme, optimal kontrol teorisi, biyoloji, yapay zeka ve daha birçok araştırma alanlarında ortaya çıkan problemlere küme-değerli dönüşümler ve onlara ait teoriler ile çözüm bulunabilmektedir. Bu çalışmada $x \in R^{n}, y \in R^{m}, y \geq 0, A$ bir $r \times n$ matris, $B$ bir $r \times m$ matris olmak üzere bir $\left(x_{0}, y_{0}\right) \in R^{n} \times R^{m}$ için $A x_{0}-$ $B y_{0} \leq 0$ eşitsizliğinin gerçekleşmesi durumunda polihedral küme-değerli dönüşümler kullanılarak $A x-B y \leq 0$ şeklinde verilen bir eşitsizlik sisteminin herhangi bir $x$ için $y$ 'ye göre çözülebilir olabilmesi için bir yeter koşul verilmektedir. Bu amaçla önce, verilen eşitsizlik sistemi uygun bir konveks küme-değerli dönüşüm ile ifade edildi sonra da o küme-değerli dönüşümün eşlenik dönüşümü belirlendi.

Anahtar Kelimeler: Polihedral küme-değerli dönüşümler, Doğrusal eşitsizlik sistemlerinin çözümleri, Eşlenik küme-değerli dönüşümler, Konveks analiz, Yeter koşullar

\begin{abstract}
The use of set-valued mappings as a basic mathematical tool in solving problems arising in various studies in scientific, technical and other academic disciplines is increasing day by day. For example, one can be found solutions with using setvalued mappings and their relaited theories to some problems that arise in nonlinear analysis, nonlinear programming, mathematical economics and management, optimal control theory, biology, artificial intelligence and many other research areas. In this work, using by polyhedral set-valued mapping we get a sufficient condition for an inequality system given as $A x-B y \leq 0$ to be solvable according to $y$ for any $x$, where $x \in \mathbb{R}^{n}, y \in \mathbb{R}^{m}, y \geq 0, A$ is an $r \times n$ matrix, $B$ is an $r \times m$ matrix and $A x_{0}-B y_{0} \leq 0$ is satisfied for a point $\left(x_{0}, y_{0}\right) \in \mathbb{R}^{n} \times \mathbb{R}^{m}$. For this purpose firstly, the given inequality system is expressed with a suitable convex set-valued mapping and then the conjugate mapping of that set-valued mapping is determined. Keywords: Polyhedral set-valued mappings, Solutions of linear inequality systems, Conjugate set-valued mappings, Convex analysis, Sufficient conditions
\end{abstract}

\section{GİRIŞ}

Bu çalışmanın amacı $x \in \mathbb{R}^{n}, y \in \mathbb{R}^{m}, y \geq 0, A$ bir $r \times n$ matris, $B$ bir $r \times m$ matris olmak üzere bir $\left(x_{0}, y_{0}\right) \in$ $\mathbb{R}^{n} \times \mathbb{R}^{m}$ için $A x_{0}-B y_{0} \leq 0$ eşitsizliğinin gerçekleşmesi durumunda $A x-B y \leq 0$ şeklinde verilen eşitsizlik sisteminin herhangi bir $x$ için $y$ 'ye göre çözülebilir olabilmesi için polihedral küme-değerli dönüşümlerin eşlenik dönüşümlerini kullanarak bir yeter koşul vermektir. Bu amaçla Pshenichnyi tarafindan [1]'de verilmiş olan yerel eşlenik küme-değerli dönüşüm kavramı yerel bir değişken barındırdığından konveks optimizasyon problemlerinde gerek ve yeter koşulları belirlemede kullanılabilmektedir. Bu kavram Rockafellar'ın [2]'de verdiği küme-değerli dönüşümlerin bir genelleştirilmişi olan "bifunction" kavramı ile yakından ilişkili olmakla beraber "bifunction" kavramı daha çok dualite problemlerinin ispatı için kullanılmaktadır. Öte yandan bir lineer programlama probleminin uygun çözümler kümesi konveks polihedral bir küme olarak yazılabildiğinden polihedral kümeler lineer programlama problemlerinde önemli bir role sahiptir. Küme değerli dönüşümlerin özel bir hali olan polihedral küme-değerli dönüşümler ve bu tip küme-değerli dönüşümleri barındıran bir takım diskret ve diferansiyel içermeli problemlerin optimizasyonu Mahmudov ve öğrencileri tarafindan $[3,4,5,6,7]$ 'de ele alınmıştır.

\section{MATERYAL VE YÖNTEM}

$\mathrm{Bu}$ bölümde konveks analizde kullanılan ve bu çalışmanın konusu olan bazı temel kavramlar tanıtılacaktır. $\mathrm{Bu}$ amaçla yalnızca çalışma içerisinde referans verilecek tanım, önerme, teorem ve sonuçlara kendi aralarında 
sıralanacak biçimde numara verilmiştir. Ayrıca daha önceden bilinen önerme, teorem ve sonuçların ispatları tekrardan kaçınmak için referans gösterilerek verilecektir. Konveks analize ait kavramlar ve sonuçlar ile ilgili derinlemesine bilgi için [2,8,9] kaynaklarına bakılmalıdır.

$\mathbb{R}$ reel sayılar kümesi olmak üzere her $i=1, \ldots, n$ için $x^{i} \in \mathbb{R}$ gerçekleyen $x=\left(x^{1}, \ldots, x^{n}\right)$ vektörlerinin $n$ boyutlu Öklid uzayı $\mathbb{R}^{n}$ ile gösterilecektir. $x_{1}, x_{2} \in \mathbb{R}^{n}$ vektörleri için iç çarpım $\left\langle x_{1}, x_{2}\right\rangle=\sum_{i=1}^{n} x_{1}^{i} x_{2}^{i}$ ve bu iç çarpım yardımıyla da bir $x \in \mathbb{R}^{n}$ vektörünün normu $\|x\|=\sqrt{\langle x, x\rangle}$ şeklinde tanımlanacaktır. $x_{1}, x_{2} \in \mathbb{R}^{n}$ vektörleri için $x_{1} \leq x_{2}$ yazıllışı ile her bir $i=1, \ldots, n$ için $x_{1}^{i} \leq x_{2}^{i} \quad$ eşitsizliklerinin gerçekleştiği ifade edilecektir. Sifir reel sayısı ile ilgili uzaydaki sıfır vektörü olan orijin aynı 0 sembolü ile gösterilecektir.

Bir $K \subseteq \mathbb{R}^{n}$ kümesi ancak ve yalnız her $x_{1}, x_{2} \in K$ ve her $0 \leq \lambda \leq 1$ için $(1-\lambda) x_{1}+\lambda x_{2} \in K$ içermesini sağlandığında konveks olarak adlandırılır. $K$ kümesinin içi int $K$, kapanışı $\bar{K}$, afin örtüsü $\operatorname{aff}(K)$ ve izafi içi ri $K$ ile gösterilecektir.

Önerme 1. [1] $\emptyset \neq K \subseteq R^{n}$ konveks bir küme olmak üzere daima $r i K \neq \varnothing$ geçerlidir.

Eğer $K \subseteq \mathbb{R}^{n}$ kümesi sonlu sayıdaki kapalı yarı-uzayın kesişimi olarak ifade edilebiliyorsa, yani $i=1,2, \ldots, r$ için $b_{i} \in R^{n}$ ve $\alpha_{i} \in R$ olmak üzere $\left\langle x, b_{i}\right\rangle \leq \alpha_{i}$ biçimindeki sonlu sayıda eşitsizlikler sisteminin çözümü ise $K$ kümesi bir polihedral küme denir. Dolayısıyla herhangi bir polihedral küme $A$ bir $r \times n$ matris ve $b$ bir $r$-boyutlu sütun vektör olmak üzere $\{x \in$ $\left.R^{n}: A x \leq b\right\}$ kümesi ile ifade edilebilir. Tanım gereği polihedral kümeler kapalı ve konveks bir kümelerdir.

Bir $\emptyset \neq K \subseteq \mathbb{R}^{n}$ kümesi her $\lambda>0$ ve her $x \in K$ için $\lambda x \in K$ içermesini gerçekliyorsa koni olarak adlandırılır. Eğer bu $K$ kümesi aynı zamanda konveks ise o zaman bu koniye konveks koni denir.

Tanım 1. Konveks bir $K$ konisinin dual konisi $K^{*}=$ $\left\{x^{*} \in \mathbb{R}^{n}: \forall x \in K\right.$ için $\left.\left\langle x, x^{*}\right\rangle \geq 0\right\}$ şeklinde tanımlanan kapalı ve konveks bir konidir.

Önerme 2. [8, 9] Herhangi bir $\emptyset \neq K \subseteq \mathbb{R}^{n}$ konveks konisi için $K^{* *}=\bar{K}$ bağıntısı geçerlidir.

$X$ ve $Y$ sonlu boyutlu Öklid uzayları ve $Z=X \times Y$ olsun. Herhangi bir $M \subseteq Z$ kümesini göz önüne alınsın. Bu durumda $M$ kümesi her $x \in X$ için $F(x)=\{y$ : $(x, y) \in M\}$ bağıntısı ile bir $F(x) \subseteq Y$ kümesi belirler. Bu $F$ bağıntısına $X$ 'ten $Y$ 'ye bir küme-değerli dönüşüm denir ve kısaca $F: X \rightarrow P(Y)$ biçiminde ifade edilir, burada $P(Y)$ ile $Y$ kümesinin tüm alt kümelerinin kümesi olan $Y$ kümesinin kuvvet kümesi gösterilmektedir. $\mathrm{Bu}$ çalışmada bundan sonra aksi söylenmedikçe $F$ dönüşümü ile $F: X \rightarrow P(Y)$ kümedeğerli dönüşümü kastedilecektir.
Bir $F$ küme-değerli dönüşümüne ilişkin aşağıdaki iki küme tanımlanır:

$$
\begin{aligned}
& \operatorname{dom} F=\{x: F(x) \neq \emptyset\} \subseteq X \text { ve } \\
& \operatorname{gph} F=\{(x, y): y \in F(x)\} \subseteq X \times Y .
\end{aligned}
$$

Dikkat edilirse $g p h F$ kümesi $F$ küme-değerli dönüşümünü tek türlü belirleyen bir kümedir.

Tanım 2. Eğer $g p h F$ kümesi $X \times Y$ 'de konveks bir küme ise $F$ dönüşümüne konveks küme-değerli dönüşüm denir. Bu durumda herhangi $x_{1}, x_{2} \in X$ ve $0 \leq \lambda \leq 1$ için aşağıdaki

$$
F\left((1-\lambda) x_{1}+\lambda x_{2}\right) \supseteq(1-\lambda) F\left(x_{1}\right)+\lambda F\left(x_{2}\right)
$$

kapsama gerçekleşir.

$\emptyset \neq K$ konveks bir küme olduğunda cone $(K)$

$=\{\lambda x: \lambda>0, x \in K\}$ şeklinde oluşturulan kümeye $K$ kümesinin doğurduğu konveks koni denir.

Tanım 3. $F$ bir konveks dönüşüm olduğunda $z_{0}=\left(x_{0}, y_{0}\right) \in g p h F$ olmak üzere

$$
\begin{aligned}
K_{g p h F}\left(z_{0}\right)= & \operatorname{cone}\left(g p h F-z_{0}\right) \\
= & \left\{(\bar{x}, \bar{y}): \bar{x}=\lambda\left(x-x_{0}\right), \bar{y}=\lambda\left(y-y_{0}\right),\right. \\
& \lambda>0,(x, y) \in g p h F\}
\end{aligned}
$$

şeklinde tanımlanan koniye konveks $g p h F$ kümesinin $z_{0} \in g p h F$ noktasındaki teğet yönler konisi denir.

$z \in g p h F$ olmak üzere $K_{g p h F}(z)$ konveks konisinin belirlediği konveks küme-değerli dönüşümü

$$
F(\bar{x} ; z)=\left\{\bar{y}:(\bar{x}, \bar{y}) \in K_{g p h F}(z)\right\}
$$

ile gösterilir $[5,8,9]$.

Tanım 4. $F$ bir konveks dönüşüm, $z \in g p h F$ ve $K_{g p h F}^{*}(z)$ konisi $K_{g p h F}(z)$ konisinin dual konisi olmak üzere

$$
F^{*}\left(y^{*} ; z\right)=\left\{x^{*}:\left(-x^{*}, y^{*}\right) \in K_{g p h F}^{*}(z)\right\}
$$

şeklinde tanımlanan küme-değerli dönüşüme $F$ dönüşümünün $z \in g p h F$ noktasındaki yerel eşlenik dönüşümü denir.

Sonuç 1. Dual koni ve yerel eşlenik dönüşüm tanımları kullanılarak $x^{*} \in F^{*}\left(y^{*} ; z\right)$ içermesinin gerçekleşmesi için gerek ve yeter koşulun her $(\bar{x}, \bar{y}) \in K_{g p h ~}(z)$ için $-\left\langle\bar{x}, x^{*}\right\rangle+\left\langle\bar{x}, y^{*}\right\rangle \geq 0$ eşitsizliğinin sağlanması olduğu sonucuna varılır.

Eğer $g p h F$ kümesi $X \times Y$ 'de polihedral bir küme ise $F$ dönüşümüne bir polihedral dönüşüm denir. Polihedral kümelerin konveks oldukları bilgisi ve Tanım 2 birlikte 
ele alındığında böyle bir $F$ dönüşümünün konveks bir dönüşüm olduğu görülür.

Teorem 1. $[5,8] x \in \mathbb{R}^{n}, y \in \mathbb{R}^{n}, A$ bir $r \times n$ matris, $B$ bir $r \times n$ matris, $A^{T}$ ve $B^{T}$ sirasiyla $A$ ve $B$ matrislerinin transpozeleri, $d$ vektörü $r$-boyutlu bir sütun vektör olmak üzere $g p h F=\{(x, y): A x-$ $B y \leq d\}$ polihedral dönüşümü verilsin. Bu durumda $g p h F$ kümesinin $z_{0} \in g p h F$ noktasındaki $K_{g p h ~}\left(z_{0}\right)$ teğet yönler konisinin dual konisi aşağıdaki konidir;

$K_{g p h F}^{*}\left(z_{0}\right)=\left\{\left(x^{*}, y^{*}\right): x^{*}=-A^{T} u, \quad y^{*}=B^{T} u\right.$,

$$
\left.0 \leq u \in \mathbb{R}^{r}\right\}
$$

\section{BULGULAR VE TARTIŞMA}

Bu bölümde $x \in \mathbb{R}^{n}, y \in \mathbb{R}^{m}$ ve $A$ bir $r \times n$ matris, $B$ bir $r \times m$ matris olmak üzere

$A x-B y \leq 0, y \geq 0$

eşitsizlik sisteminin herhangi bir $x$ için y’ye göre çözülebilirliği problemi küme-değerli dönüşüm kavramını kullanarak ele alınacak ve eşlenik kümedeğerli dönüşüm yardımıyla çözülebilirliği için bir yeter koşul verilecektir.

Şimdi (5) sistemine karşılık gelen aşağıdaki $F: \mathbb{R}^{n} \rightarrow$ $P\left(\mathbb{R}^{m}\right)$ küme-değerli dönüşümü tanımlayalım,

$$
F(x)=\{y: A x-B y \leq 0, y \geq 0\}
$$

$\mathrm{Bu}$ durumda (5) sisteminin çözülebilirliği problemi $F(x) \neq \varnothing \quad$ sağlanması durumunda koşulların belirlenmesine problemine indirgenmiş olur.

Önerme 3. $F(\bar{x} ; z)=\left\{\bar{y}:(\bar{x}, \bar{y}) \in K_{g p h F}(z)\right\}$ şeklinde tanımlanan bir küme-değerli dönüşüm $\bar{x}$ 'ye göre pozitif homojendir.

İspat. $\lambda>0$ olmak üzere Eşitlik (3) kullanılarak

$$
\begin{aligned}
F(\lambda \bar{x} ; z) & =\left\{\bar{y}:(\lambda \bar{x}, \bar{y}) \in K_{g p h F}(z)\right\} \\
& =\left\{\bar{y}: \lambda\left(\bar{x}, \frac{\bar{y}}{\lambda}\right) \in K_{g p h F}(z)\right\} \\
& =\left\{\bar{y}:\left(\bar{x}, \frac{\bar{y}}{\lambda}\right) \in \frac{1}{\lambda} K_{g p h F}(z)\right\} \\
& \stackrel{*}{=}\left\{\bar{y}:\left(\bar{x}, \frac{\bar{y}}{\lambda}\right) \in K_{g p h F}(z)\right\} \\
& =\left\{\lambda \bar{y}:(\bar{x}, \bar{y}) \in K_{g p h F}(z)\right\} \\
& =\lambda\left\{\bar{y}:(\bar{x}, \bar{y}) \in K_{g p h F}(z)\right\} \\
& =\lambda F(\bar{x} ; z)
\end{aligned}
$$

bulunur. Burada, $K_{g p h F}(z)$ kümesi bir koni olduğundan $\lambda>0$ için $\frac{1}{\lambda} K_{g p h F}(z)=K_{g p h ~}(z)$ eşitliğ sağlanacağ ${ }_{1}$ için $(*)$ eşitliği geçerlidir.

Sonuç 2. $\operatorname{dom} F(\cdot ; z)$ kümesi $\mathbb{R}^{n}$ 'de konveks bir konidir.

İspat. $g p h F(\cdot ; z)=K_{g p h F}(z)$ ve $K_{g p h F}(z)$ konveks olduğu için Tanım 2 nedeniyle $F(\cdot ; z)$ konveks bir küme-değerli dönüşümdür. Bu durumda $\operatorname{dom} F(\cdot ; z)$ kümesi $\mathbb{R}^{n}$ 'de konveks bir kümedir. Çünkü $0 \leq \lambda \leq 1$ ve $\bar{x}_{1}, \bar{x}_{2} \in \operatorname{dom} F(\cdot ; z)$ için Eşitlik (1) nedeniyle $F\left(\lambda \bar{x}_{1}+(1-\lambda) \bar{x}_{2} ; z\right) \supseteq \lambda F\left(\bar{x}_{1} ; z\right)+(1-\lambda) F\left(\bar{x}_{1} ; z\right) \neq \emptyset$ geçerlidir. Üstelik $\bar{x} \in \operatorname{dom} F(\cdot ; z)$ ve $\lambda>0$ için $\lambda \bar{x} \in$ $\operatorname{dom} F(\cdot ; z)$ gerçekleşir. Çünkü $\bar{x} \in \operatorname{dom} F(\cdot ; z)$ ise $F(\bar{x} ; z) \neq \emptyset$ olup Önerme 3 nedeniyle $F(\lambda \bar{x} ; z)=$ $\lambda F(\bar{x} ; z) \neq \emptyset$ gerçekleşir bu ise $\lambda \bar{x} \in \operatorname{dom} F(\cdot ; z)$ demektir. Şu halde $\operatorname{dom} F(\cdot ; z)$ kümesi $\mathbb{R}^{n}$ 'de konveks bir konidir.

Önerme 4. $(\operatorname{dom} F(\cdot ; z))^{*}=-F^{*}(0 ; z)$ geçerlidir.

İspat. $\operatorname{dom} F(\cdot ; z)$ kümesinin Sonuç 2 nedeniyle $\mathbb{R}^{n}$ 'de konveks bir koni olduğu göz önüne alınırsa aşağıda hesaplanan dual konisinden söz edilebilir. Bu durumda Tanım 1 ve Sonuç 1 yardımıyla

$(d o m F(\cdot ; z))^{*}$

$=\left\{x^{*}: \forall \bar{x} \in \operatorname{dom} F(\cdot ; z) \operatorname{için}\left\langle\bar{x}, x^{*}\right\rangle \geq 0\right\}$

$=\left\{x^{*}: \forall(\bar{x}, \bar{y}) \in K_{\text {gph } F}(z) \operatorname{için}\left\langle\bar{x}, x^{*}\right\rangle+\langle\bar{y}, 0\rangle \geq 0\right\}$

$=\left\{x^{*}:\left(x^{*}, 0\right) \in K_{g p h F}^{*}(z)\right\}$

$=-\left\{-x^{*}:\left(x^{*}, 0\right) \in K_{g p h F}^{*}(z)\right\}$

$=-\left\{x^{*}:\left(-x^{*}, 0\right) \in K_{g p h F}^{*}(z)\right\}$

$=-F^{*}(0 ; z)$

elde edilir.

Önerme 5. $(\operatorname{dom} F(\cdot ; z))^{*}=\{0\}$ ise $\operatorname{dom} F(\cdot ; z)=$ $\mathbb{R}^{n}$ olur.

İspat. $K:=\operatorname{dom} F(\cdot ; z)$ diyelim. Sonuç 2 nedeniyle $K \subseteq \mathbb{R}^{n}$ konveks konidir. Dolayisıyla Önerme 2 yardımiyla $\bar{K}=K^{* *}=\{0\}^{*}=\mathbb{R}^{n}$ elde edilir. Yani $K$ konveks konisi $\mathbb{R}^{n}$ uzayında yoğundur. Şimdi iddiamız $K=\mathbb{R}^{n} \quad$ olduğudur. Bunun için $\mathbb{R}^{n} \subseteq$ $K$ gerçeklendiğini göstermek yeterlidir. Eğer bu kapsama geçerli olmasayd $\bar{x}_{0} \notin K$ gerçekleyen bir $\bar{x}_{0} \in \mathbb{R}^{n}$ elemanı var olurdu. Öte yandan $K$ bir konveks koni ve $\bar{K}=\mathbb{R}^{n}$ olduğu için konveks analiz kavramları yardımıyla $K$ konisinin afin örtüsünün $\operatorname{aff}(K)=\mathbb{R}^{n}$ olduğu görülür. $\mathrm{Bu}$ durumda $\mathrm{K}$ konveks konisinin içi ile izafi içi çakışır, yani int $K=$ ri $K$ olur. Önerme 1 nedeniyle konveks $\mathrm{K}$ kümesinin izafi içi boş değildir. Böylece $\bar{x}_{1} \in$ int $K(\neq \emptyset)$ olmak üzere $\hat{x}:=2 \bar{x}_{0}-\bar{x}_{1}$ noktasını tanımlayabiliriz. $K$ konveks konisi $\mathbb{R}^{n}$ uzayında yoğun olduğundan $\forall k \in \mathbb{N}$ için $\hat{x}_{k} \in K$ ve $\lim _{k \rightarrow \infty} \hat{x}_{k}=\hat{x}$ koşulunu sağlayan bir $\left(\hat{x}_{k}\right)$ dizisi vardır. Şimdi $\forall k \in \mathbb{N}$ için elemanları

$\bar{x}_{k}=2 \bar{x}_{0}-\hat{x}_{k}$

şeklinde tanımlanan $\left(\bar{x}_{k}\right)$ dizisini göz önüne alalım. $\lim _{k \rightarrow \infty} \bar{x}_{k}=\lim _{k \rightarrow \infty} 2 \bar{x}_{0}-\hat{x}_{k}=2 \bar{x}_{0}-\hat{x}=2 \bar{x}_{0}-\left(2 \bar{x}_{0}-\right.$ $\left.\bar{x}_{1}\right)=\bar{x}_{1}$ bulunur. Bu durumda $\bar{x}_{1} \in$ int $K$ olduğundan yeterince büyük $k \in \mathbb{N}$ doğal sayıları için $\bar{x}_{k} \in K$ olur. 
Sonuç olarak Eşitlik (6)'dan $\bar{x}_{0}=\frac{1}{2} \underbrace{\bar{x}_{k}}_{\in K}+\frac{1}{2} \underbrace{\hat{x}_{k}}_{\in K}$ ve $K$ konveks olduğundan $\bar{x}_{0} \in K$ bulunur ki bu durum $\bar{x}_{0} \notin$ $K$ olması ile çelişir. Dolayısıyla $R^{n} \subseteq K$ kapsaması geçerlidir ve sonuçta $K=\operatorname{dom} F(\cdot ; z)=\mathbb{R}^{n}$ bulunur.

Teorem 2. $F$ bir konveks küme-değerli dönüşüm olmak üzere $z=\left(x_{0}, y_{0}\right) \in g p h F$ ve $F^{*}(0 ; z)=\{0\}$ gerçekleşsin. Bu durumda öyle bir $\delta>0$ sayısı vardır ki $\|\bar{x}\|<\delta$ sağlayan her $\bar{x} \in R^{n}$ için $F\left(x_{0}+\bar{x}\right) \neq \emptyset$ olur.

İspat. $F^{*}(0 ; z)=\{0\}$ olduğundan Önerme 4 nedeniyle $(\operatorname{dom} F(\cdot ; z))^{*}=\{0\}$ olur, bu durumda Önerme 5 nedeniyle $\operatorname{dom} F(\cdot ; z)=\mathbb{R}^{n}$ bulunur. Herhangi bir $\bar{x} \in \mathbb{R}^{n}$ verilsin. $\mathrm{Bu}$ durumda $x \in \operatorname{dom} F(\cdot ; z)$ olacağından $(\bar{x}, \bar{y}) \in K_{g p h F}(z)$ içermesini sağlayan bir $\bar{y}$ noktası vardır. Dolayısıyla Tanım 3'teki Eşitlik (2) yardımıla $\bar{x}=\gamma\left(x-x_{0}\right)$ ve $\bar{y}=\gamma\left(y-y_{0}\right)$ olacak biçimde bir $\gamma>0$ sayısı ve bir $(x, y) \in g p h F$ elemanı vardır. Eğer $\delta=\frac{1}{\gamma}>0$ olarak seçilirse $0 \leq \lambda \leq \delta$ sağlayan her $\lambda$ için $g p h F$ kümesinin konveks olması ve $0 \leq \lambda \gamma \leq 1$ nedeniyle

$\left(x_{0}+\lambda \bar{x}, y_{0}+\lambda \bar{y}\right)=\left(x_{0}+\lambda \gamma\left(x-x_{0}\right), y_{0}+\lambda \gamma(y-\right.$ $\left.\left.y_{0}\right)\right)=\left((1-\lambda \gamma) x_{0}+\lambda \gamma x,(1-\lambda \gamma) y_{0}+\lambda \gamma y\right) \in g p h F$

içermesi gerçekleşir. $\mathrm{Bu}$ ise $F\left(x_{0}+\lambda \bar{x}\right) \neq \varnothing$ olması demektir. Öte yandan $i=1,2, \ldots, n+1$ olmak üzere öyle uygun $\tilde{x}_{i} \in \mathbb{R}^{n}$ vektörleri bulunabilir ki $S=$ $\left\{\lambda_{1} \tilde{x}_{1}+\lambda_{2} \tilde{x}_{2}+\cdots+\lambda_{n+1} \tilde{x}_{n+1}: \lambda_{i} \geq 0\right.$ ve $\sum_{i=1}^{n+1} \lambda_{i}=$ $1\}$ şeklinde oluşturulan konveks küme (bu tür kümelere $n$-boyutlu simpleks denir) orijini bir iç nokta olarak içerir $\left[10\right.$, s.123]. Yani $\mathbb{R}^{n}$ de öyle $n+1$ tane $\tilde{x}_{i}$ vektörleri bulunabilir ki bu vektörlerin konveks örtüsü olan $S$ konveks kümesi için $0 \in$ int $S$ gerçekleşir. Bu durumda bu vektörlerin boyları istenildiği kadar kısaltılsa da $0 \in$ int $S$ sağlanır. Dolayısıyla $i=$ $1,2, \ldots, n+1$, olmak üzere $0<\lambda_{i}<\delta$ şeklinde seçilen uygun $\lambda_{i}$ sayıları yardımıyla tanımlanan $\bar{x}_{i}=$ $\lambda_{i} \tilde{x}_{i} \quad$ vektörleri için $F\left(x_{0}+\bar{x}_{i}\right) \neq \varnothing$ gerçekleşir. Böylece orijinin $\delta$ komşuluğundaki her $\bar{x}$ noktası yani $\|\bar{x}\|<\delta$ sağlayan her $\bar{x}$ noktası, $\lambda_{i} \geq 0$ ve $\sum_{i=1}^{n+1} \lambda_{i}=$ 1 olmak üzere $\bar{x}=\lambda_{1} \bar{x}_{1}+\lambda_{2} \bar{x}_{2}+\cdots+\lambda_{n+1} \bar{x}_{n+1}$ biçiminde ifade edilebilir. $\mathrm{Bu}$ durumda $\|\bar{x}\|<\delta$ sağlayan her $\bar{x}$ noktası için Tanım 2'deki Eşitlik (1) kullanılarak

$$
F\left(x_{0}+\bar{x}\right)=F\left(\sum_{i=1}^{n+1} \lambda_{i}\left(x_{0}+\bar{x}_{i}\right)\right) \supseteq \sum_{i=1}^{n+1} \lambda_{i} F\left(x_{0}+\bar{x}_{i}\right) \neq \varnothing
$$

elde edilir.

Sonuç 3. $x \in \mathbb{R}^{n}, y \in \mathbb{R}^{m}$ ve $A$ bir $r \times n$ matris, $B$ bir $r \times m$ matris, $U=\left\{u \geq 0: B^{T} u \leq 0\right\}$ olmak üzere $U \subseteq \operatorname{Ker} A^{T}$ gerçekleşsin. Bu durumda $A x-B y \leq 0$, $y \geq 0$ eşitsizlik sistemi herhangi bir $x$ için y'ye göre çözülebilirdir. Burada $A^{T}$ ile $A$ matrisinin transpozu ve $\operatorname{Ker} A$ ile de $A$ matrisinin çekirdeği gösterilmektedir.
İspat. Grafiği $g p h F=\{(x, y): A x-B y \leq 0, y \geq 0\}$ olan polihedral küme-değerli $F$ dönüşümünü göz önüne alalım. gph $F$ kümesi tanımı gereği $\mathbb{R}^{n+m}$ uzayında polihedral bir küme olduğu için $F$ dönüşümü konvekstir. $0:=(0,0) \in g p h F$ olduğu açıktır. Dikkat edilirse Teorem 2'nin ifadesinde bulunan $F^{*}(0 ; z)$ ifadesi Eşitlik (4)'teki $y^{*}=0$ haline denk gelmektedir. Dolayısıyla $x_{0}=0, y_{0}=0$ ve Eşitlik (4)'te $y^{*}=0$ alınırsa Teorem 2'nin koşulları sağlanır. Öte yandan Teorem 1 'de $z_{0}=(0,0)$ ve $y \in \mathbb{R}^{m}$ olarak alınırsa $K_{\text {gph } F}\left(z_{0}\right)$ konisinin dual konisi

$$
\begin{aligned}
K_{g p h F}^{*}(0)= & \left\{\left(x^{*}, y^{*}\right): x^{*}=-A^{T} u, y^{*}=B^{T} u+v,\right. \\
& \left.0 \leq u \in R^{r}, 0 \leq v \in R^{m}\right\} \\
= & \left\{\left(-A^{T} u, B^{T} u+v\right): 0 \leq u \in R^{r},\right. \\
& \left.0 \leq v \in R^{m}\right\}
\end{aligned}
$$

olarak hesaplanır. Bu durumda Tanım 4'teki Eşitlik (4) gereği $F^{*}\left(y^{*} ; 0\right)=\left\{A^{T} u: y^{*}=B^{T} u+v, 0 \leq u \in\right.$ $\left.\mathbb{R}^{r}, 0 \leq v \in \mathbb{R}^{m}\right\}$ olur. O halde Teorem 2 nedeniyle $F^{*}(0 ; 0)=\{0\}$ koşulunun gerçekleşmesi, $B^{*} u \leq 0$ koşulunun sağlayan $0 \leq u$ vektörlerinin $A^{T} u=0$ eşitliğini sağlamasına denk olur. Başka bir deyişle $A x-B y \leq 0, y \geq 0$ eşitsizlik sisteminin herhangi bir $x \in \mathbb{R}^{n}$ için y'ye göre çözülebilir olması için $U=$ $\left\{u \geq 0: B^{T} u \leq 0\right\} \quad$ olmak üzere $U \subseteq \operatorname{Ker} A^{T}$ kapsamasının gerçekleşmesi yeterli olur.

\section{SONUÇ VE DEĞERLENDİRMELER}

Bu çalışmada $x \in \mathbb{R}^{n}, y \in \mathbb{R}^{m}, y \geq 0, A$ bir $r \times n$ matris, $B$ bir $r \times m$ matris olmak üzere bir $\left(x_{0}, y_{0}\right) \in$ $\mathbb{R}^{n} \times \mathbb{R}^{m} \quad$ için $\quad A x_{0}-B y_{0} \leq 0 \quad$ eşitsizliğinin gerçekleşmesi durumunda $A x-B y \leq 0$ şeklinde verilen eşitsizlik sisteminin herhangi bir $x$ için $y$ 'ye göre çözülebilirliği problemi ele alındı. Bu problem, önce $[3,5,8]$ 'de incelenen polihedral küme-değerli dönüşümler yardımıyla farklı bir biçimde ifade edildi, sonra da [1]'de verilmiş olan yerel eşlenik kümedeğerli dönüşüm kavramı kullanılarak Sonuç 3 'te ifade edilen bir yeter koşul verildi. Benzer yöntem kullanılarak farklı eşitlik ya da eşitsizlik sistemlerinin çözülebilirliği veya daha genel olarak bir kapalı fonksiyon yardımıyla verilmiş bir denklemin bir noktanın komşuluğunda çözülebilirliği için yeter koşullar tespit edilebilir.

\section{TESEKKÜR}

$\mathrm{Bu}$ çalışmanın gerçekleşmesini teşvik eden Prof. Dr. Elimhan Mahmudov'a ve kıymetli eleştirileriyle makaleyi değerlendirerek son halini almasını sağlayan hakemlere teşekkür ederim.

\section{KAYNAKLAR}

[1] Pshenichnyi, B.N. (1972). Convex multivalued mappings and their conjugates. Kibernetika, 3, 94-102.

[2] Rockafellar, R.T. (1970). Convex analysis. Princeton University Press. 
[3] Mahmudov, E.N. and Psenichnyi, B.N. (1979). Polyhedral mappings. Izvestija Akademii Nauk Azerbaidzanskoi SSR. Serija Fiziko-Tehniceskih i Matematiceskih, Nauk, 2, 10-15.

[4] Mahmudov, E.N. and Değer, Ö. (2005). On an optimization problem described by multivalued mappings and duality. Applied and Computational Mathematics an International Journal, 4(2),192-199.

[5] Değer, Ö. (2009). Polihedral dahil etmelerde optimallik için gerek ve yeter koşullar. Doktora Tezi, İstanbul Üniversitesi, Türkiye.

[6] Değer, Ö. (2011). On optimality conditions for a convex optimization problem with polyhedral discrete inclusions. Ístanbul Üniversitesi Fen
Fakültesi Matematik Fizik Astronomi Dergisi, (N.S.) 3 (2008/09), 109-118.

[7] Mahmudov, E.N., Demir, S. and Değer, Ö. (2016). Optimization of third-order discrete and differential inclusions described by polyhedral set-valued mappings. Applicable Analysis, An International Journal, 95(9), 1831-1844.

[8] Mahmudov, E.N. (2011). Approximation and optimization of discrete and differential inclusions. Elsevier.

[9] Pshenichnyi, B.N. (1980). Convex analysis and extremal problems. Nauka, Moscow, (Russian).

[10] Gamkrelidze, R.V. (1978). Principles of optimal control theory. Mathematical Concepts and Methods in Science and Engineering 7, Springer US. 